\title{
Comparison of single and multiple sessions of percutaneous sclerotherapy for simple renal cyst
}

\author{
B.H. CHUNG, J.H. KIM, C.H. HONG, S.C. YANG and M.S. LEE \\ Department of Urology, Yonsei University College of Medicine, Seoul, Korea
}

Objective To compare the results of single and repeated percutaneous sclerotherapy in patients with simple renal cysts.

Patients and methods Eighty-two patients with simple renal cysts underwent needle aspiration and sclerotherapy under ultrasonographic guidance. Forty-two patients (group 1) underwent one session of sclerotherapy with 99\% ethanol immediately after aspiration and 40 patients (group 2) underwent sclerotherapy at least twice. The patients were followed up using ultrasonography at 3month intervals. The complete disappearance or a reduction of more than half in the diameter of the renal cyst was considered a successful treatment.

Results The mean diameter of the renal cysts was not significantly different in group $1(6.12 \mathrm{~cm})$ and 2 $(6.75 \mathrm{~cm})$. There was complete or partial regression in eight (19\%) and 16 (38\%) in group 1, and in $29(73 \%)$ and nine $(23 \%)$ in group 2 , respectively. The overall success rate was significantly better in group 2 (95\%) than in group $1(57 \% ; P<0.001)$.

Conclusions These results suggest that multiple sclerotherapy is better than a single injection of sclerosant for reducing the recurrence of simple renal cysts.

Keywords Simple renal cyst, percutaneous sclerotherapy

\section{Introduction}

Although simple renal cysts are benign and usually require no treatment, intervention is needed when symptoms and/ or urinary tract obstruction are associated with the cyst. Simple aspiration or sclerotherapy of simple renal cysts are minimally invasive procedures with little morbidity, frequently undertaken to treat these patients. However, the recurrence rate after simple aspiration alone is $41-78 \%$ [1-4] and can reach $32-100 \%$ after a single sclerotherapy session using ethanol $[4,5]$. The recurrence rate may be high even after sclerotherapy because patients undergo only one sclerotherapy session. We compared the results of single and repeated sessions of percutaneous sclerotherapy to determine whether multiple sclerotherapy can reduce the recurrence rate in patients with simple renal cysts.

\section{Patients and methods}

Between March 1990 and September 1997, 82 patients (mean age 54.8 years, range 41-72) with 82 simple renal cysts underwent sclerotherapy using 99\% ethanol. The indications for treatment were flank/back pain (34 patients), haematuria (four), compression of the urinary collecting system (21) and/or a cyst diameter of $>5 \mathrm{~cm}$ regardless of the presence of symptoms (23 patients). A

Accepted for publication 9 November 1999 simple renal cyst was diagnosed when it satisfied the criteria of Bosniak [5].

Of the 82 patients, 42 received a single session sclerotherapy immediately after aspiration (group 1) and 40 underwent sclerotherapy at least twice after a $6 \mathrm{~F}$ pigtail catheter was placed in the renal cyst (group 2). The same three patients in groups 1 and 2 underwent repeated sclerotherapy after having undergone unsuccessful singlesession sclerotherapy. The patients were followed up with ultrasonography at 3-month intervals. The disappearance of symptoms and of the renal cyst were considered to be complete regression, and the relief of symptoms with a reduction in the diameter of the cyst by more than half as partial regression. Treatment was considered to have failed when the cyst recurred to more than half the diameter before treatment and/or when the symptoms persisted.

For single-session sclerotherapy (group 1), patients were placed prone and after confirming the location of the renal cyst using ultrasonography, the selected puncture site was infiltrated with $2 \%$ lidocaine. An $18 \mathrm{G}$ puncture needle was placed in the cyst under ultrasonographic guidance. The volume of the fluid aspirated was recorded and the fluid sent for bacteriological, cytological and biochemical (lipid, protein, lactate dehydrogenase) tests. The shape of the cyst and the presence of extravasation or connection with the renal collecting system were evaluated after injecting the aspirated cyst with contrast medium (diatrizoate sodium, $30 \% \mathrm{w} / \mathrm{v}$ ) and normal saline in a volume equal to that of 
the aspirated fluid (final concentration, 15\%). After confirming a smooth margin and no extravasation, the contrast medium was completely drained and $25 \%$ of the aspirated volume replaced with $99 \%$ ethanol; this was completely removed after 20-30 min

For multiple-session sclerotherapy (group 2), after placing a puncture needle as in group 1, a $0.96 \mathrm{~mm} \mathrm{J-tip}$ guidewire was passed into the cyst. The puncture needle was removed and a $6 \mathrm{~F}$ pigtail catheter inserted into the renal cyst over the guidewire; subsequent procedures were then performed as described for group 1. Repeat sessions were performed at $\approx 12 \mathrm{~h}$ intervals on the same night and the next morning. The catheter was left open for natural drainage between sessions and the correct positioning of the catheter ensured by irrigating with $10-20 \mathrm{~mL}$ normal saline before each repeat session. Sclerotherapy was not carried out if the test irrigation failed or symptoms such as fever or pain developed. The pigtail catheter was removed after the final session. Student's $t$-test was used to assess any differences, with $P<0.05$ considered to indicate significance.

\section{Results}

The mean follow-up for groups 1 and 2 were 12.9 and 15.4 months, respectively. The mean diameters of the renal cysts in group $1(6.12 \mathrm{~cm})$ and $2(6.75 \mathrm{~cm})$ were not significantly different. In all cases the cyst fluid was clear, there was no abnormality on bacteriological, cytological or biochemical studies, and the renal cystogram showed no leakage, filling defect or connection with the collecting system. All patients in group 2 received two or three (mean 2.7) treatment sessions, depending on the success of the test irrigation and the presence of symptoms. There was a small amount of fluid drained after each injection and this did not differ in volume.

The complete and partial regression rates were 19\% (eight) and 38\% (16) for group 1 and 73\% (29) and 23\% (nine) for group 2, respectively. The overall success rate was significantly better in group $2(95 \%)$ than in group 1 (57\%; $P<0.001)$. Of the 28 symptomatic patients in group 1, the symptoms resolved all except eight, in whom they recurred. In group 2, 20 of 22 symptomatic patients had relief after therapy. Ten patients, including the two with a recurrent cyst in group 2, developed transient mild flank pain or fever requiring no active treatment after the second session, and underwent two sclerotherapy sessions. There were no other serious complications after therapy in either group.

\section{Discussion}

Percutaneous sclerotherapy is usually used as the first-line treatment before surgical or laparoscopic decompression of simple renal cysts that require treatment. It is clearly better than aspiration alone, where the cyst fluid almost always re-accumulates [3,4]. Of the various sclerosants, 99\% ethanol was used in the present patients because it rapidly (1-3 min) coagulates the cells lining the cyst but slowly penetrates the fibrous capsule (4-12 h), causing minimal local or systemic complications [1].

The present success rates of $57 \%$ and $95 \%$ after single and repeated sclerotherapy, respectively, are comparable with the results of Hanna and Dahniya [4], where treatment was successful in $68 \%$ and $100 \%$ of patients, respectively. The recurrence of renal cysts after sclerotherapy is probably caused by incomplete ablation of the cyst wall [1]. Therefore, it is likely that the longer contact time between ethanol and the renal cyst epithelium in group 2 contributed to the higher success rate. That the two patients who had recurrent cysts in group 2 received only two sessions also supports this view.

A small calibre pigtail catheter was used in the present patients, with no tract dilatation, and there was no damage to the collecting system or adjacent organs. Mild and transient fever and flank discomfort were the only minor complications. Therefore, multiple sclerotherapy sessions are a safe and effective treatment for simple renal cysts, further reducing the recurrence rate compared with that from single-session sclerotherapy. Furthermore, as shown in the present three patients who subsequently required multiple sessions of sclerotherapy, repeat sclerotherapy can be used before proceeding to percutaneous endoscopic, laparoscopic, or open surgical therapy after failure of simple aspiration or a single sclerotherapy session.

\section{References}

1 Bean WJ. Renal cysts: treatment with alcohol. Radiology 1981; 138: 329-31

2 Stevenson JJ, Sherwood T. Conservative management of renal masses. Br J Urol 1971; 43: 646-7

3 Raskin MM, Poole DO, Roen SA, Viamonte M Jr. Percutaneous management of renal cysts: results of a fouryear study. Radiology 1975; 115: 551-3

4 Hanna RM, Dahniya MH. Aspiration and sclerotherapy of symptomatic simple renal cysts: value of two injections of a sclerosing agent. Am J Roentgenol 1996; 167: 781-3

5 Bosniak MA. The current radiological approach to renal cysts. Radiology 1985; 158: 6

\section{Authors}

B.H. Chung, MD, PhD, Assistant Professor.

J.H. Kim, MD, Resident.

C.H. Hong, MD, Resident.

S.C. Yang, MD, PhD, Professor.

M.S. Lee, MD, PhD, Professor.

Correspondence: Byung Ha Chung, Department of Urology, Yonsei University College of Medicine, CPO Box 8044, 134 Sinchon-dong, Seodaemun-ku, Seoul, Korea 120-752.

E-mail: chung646@yumc.yonsei.ac.kr 